\title{
Interaction of thiacloprid with bovine hemoglobin using spectroscopic and molecular modeling methods
}

\author{
Chang-Yun Chen ${ }^{\mathrm{a}, \mathrm{c}}$, Bo Zhao ${ }^{\mathrm{b}, *}$ and Zheng-Wu Wang ${ }^{\mathrm{a}, *}$ \\ ${ }^{a}$ BorLuh Food Safety Center, Department of Food Science and Technology, School of Agriculture and \\ Biology, Shanghai Jiao Tong University, Shanghai, China \\ ${ }^{\mathrm{b}}$ School of Chemical and Environmental Science, Nanjing Normal University, Nanjing, China \\ ${ }^{\mathrm{c}}$ School of Biochemical and Environmental Engineering, Nanjing Xiaozhuang University, Nanjing, \\ China
}

\begin{abstract}
The interaction of thiacloprid (TL) to bovine hemoglobin (BHb) under physiological conditions was investigated by using fluorescence spectroscopy, circular dichroism spectroscopy (CD) and molecular modeling. The fluorescence intensity of $\mathrm{BHb}$ decreased regularly with the gradual increasing concentration of TL. It is observed that there was a prominent interaction between TL and BHb. The binding constants $K_{\mathrm{A}}$ at 288, 298 and $308 \mathrm{~K}$ obtained are 8.04, 5.26 and $3.08 \times 10^{4} 1 \cdot \mathrm{mol}^{-1}$, respectively. The standard enthalpy change $\left(\Delta H^{0}\right)$ and the standard entropy change $\left(\Delta S^{0}\right)$ are calculated to be $-34.54 \mathrm{KJ} \cdot \mathrm{mol}^{-1}$ and $-25.77 \mathrm{~J} \cdot \mathrm{mol}^{-1} \cdot \mathrm{K}^{-1}$, which indicated that hydrogen bonding forces play major role in the interaction between TL and $\mathrm{BHb}$. The alternations of protein secondary structure in the presence of TL were determined by CD spectroscopy. The results revealed that the content of $\alpha$-helix was decreased from $51.85 \%$ in free BHb to $48.14 \%$ in TL-BHb complex. Molecular modeling study and our experimental results both showed that the binding mode of TL-BHb complex could be attributed to hydrogen bonding and hydrophobic interaction.
\end{abstract}

Keywords: Interaction, thiacloprid, bovine hemoglobin, spectroscopic techniques, molecular modeling methods

\section{Introduction}

Chemicals released from agriculture or industry may potentially develop toxic effects in the environment and ecological systems. Among them, pesticides are actively applied and globally used for crop control and to prevent damage to plants, animals, humans or aliments. Thiacloprid (TL) [5], a relatively new neonicotinoid insecticide ((Z)-3-(6-chloro-3-pyridylmethyl)-1,3-thiazolidin-2-ylidenecyanamide) has been produced by Bayer Crop Science since 2003. It acts as an agonist on the nicotinoid acetylcholine receptor (nAChR). Neonicotinoids are systemic insecticides and enter the body via contact and ingestion. They act on the central nervous system of the insects and are mainly used in pest management against biting and sucking insects. At physiological pH levels, neonicotinoids are not protonated. As a

\footnotetext{
${ }^{*}$ Corresponding authors: Zheng-Wu Wang and Bo Zhao, BorLuh Food Safety Center, Department of Food Science and Technology, School of Agriculture and Biology, Shanghai Jiao Tong University, Shanghai 200240, China and School of Chemical and Environmental Science, Nanjing Normal University, Nanjing 210097, China. E-mails: professorwzw@ 126.com; zbchem@126.com.
} 
result of this, they have little affinity to the nAChR of vertebrates. Therefore, they generally show low acute and chronic toxicity to mammals, birds and fish. However, among neonicotinoids investigated so far thiacloprid was found to exhibit a comparatively high level of acute toxicity to fish.

Hemoglobin $(\mathrm{Hb})$ is one of the most significant proteins in the blood plasma of higher organisms. $\mathrm{Hb}$ is well known for its function in the blood circulatory system that working as a transporter of oxygen. $\mathrm{So}, \mathrm{BHb}$ was usually treated as a model protein to explore the binding properties of drugs [1].

It is necessary to investigate the interaction of TL with protein, as it can illustrate the nature of TLprotein complex in vitro and provide important insight for the understanding of the metabolism and distribution of TL in vivo, molecular functional design [3].

\section{Materials and methods}

\subsection{Materials}

Bovine hemoglobin (BHb, $\geqslant 95 \%$ purity) was purchased from Sigma Chemical Company and used without further purification. The BHb solution $(5 \mu \mathrm{M})$ was prepared in Tris-HCl buffer solution ( $\mathrm{pH} 7.4)$, and kept in the dark at $277 \mathrm{~K}$. TL was obtained from the National Institute for Control of Pharmaceutical and Bioproducts, China. The stock solution was prepared in methanol because thiacloprid easily dissolve in the methanol with a concentration of $5 \mathrm{mM}$. The buffer solution ( $\mathrm{pH} 7.4)$ consists of Tris $\left(0.2 \mathrm{~mol} \cdot \mathrm{1}^{-1}\right)$ and $\mathrm{HCl}\left(0.1 \mathrm{~mol} \cdot \mathrm{l}^{-1}\right)$. All reagents were of analytical reagent grade and distilled water was used throughout the experiment.

\subsection{Apparatus and methods}

Fluorescence spectra was recorded on a LS 50B spectrofluorimeter (PerkinElmer, USA). A slit width of $5 \mathrm{~nm} / 5 \mathrm{~nm}$ was typically used $3.0 \mathrm{ml}$ solution containing appropriate concentration of BHb $(5.0 \mu \mathrm{M})$ was titrated manually by successive additions of $5 \mathrm{mM}$ solution of TL. The $\mathrm{pH}$ measurements were carried out on a pHS-3C Exact Digital pH meter (Shanghai Leici, China). The emission spectra were obtained in the range from 300 to $500 \mathrm{~nm}$, with the excitation wavelength at $280 \mathrm{~nm}$. Circular dichroism were performed on a Jasco-20 spectropolarimeter (JASCO, Japan), using a $1 \mathrm{~mm}$ quartz cell at room temperature with a scanning speed of $20 \mathrm{~nm} / \mathrm{min}$. The spectra were recorded in the far-UV region from 200-250 nm and the buffer solution was selected as the blank and automatically subtracted during scanning. Each spectrum was the average of four scans.

Protein-ligand docking modeling was performed using Discovery Studio 2.1 software where the $\mathrm{pH}$ is set to 7.4. The crystal structure of Bhb (PDB entry code 1G09) was downloaded from the Protein Data Bank and the potential of the 3D structure of Bhb was assigned to the CHARMm force field. The structure of TL was built in Discovery Studio 2.1 and optimized by applying the CHARMm force field. During the docking process, a maximum of 10 conformers have been chosen for the final energy minimization. The conformation with the lowest energy was selected for final analysis.

\section{Results and discussion}

\subsection{Analysis of fluorescence quenching of $B H b$ by $T L$}

Fluorescence measurements were carried out to investigate whether TL interacts with BHb. Figure 1 shows the fluorescence emission spectra of BHb in the absence and presence of TL. BHb has a strong 


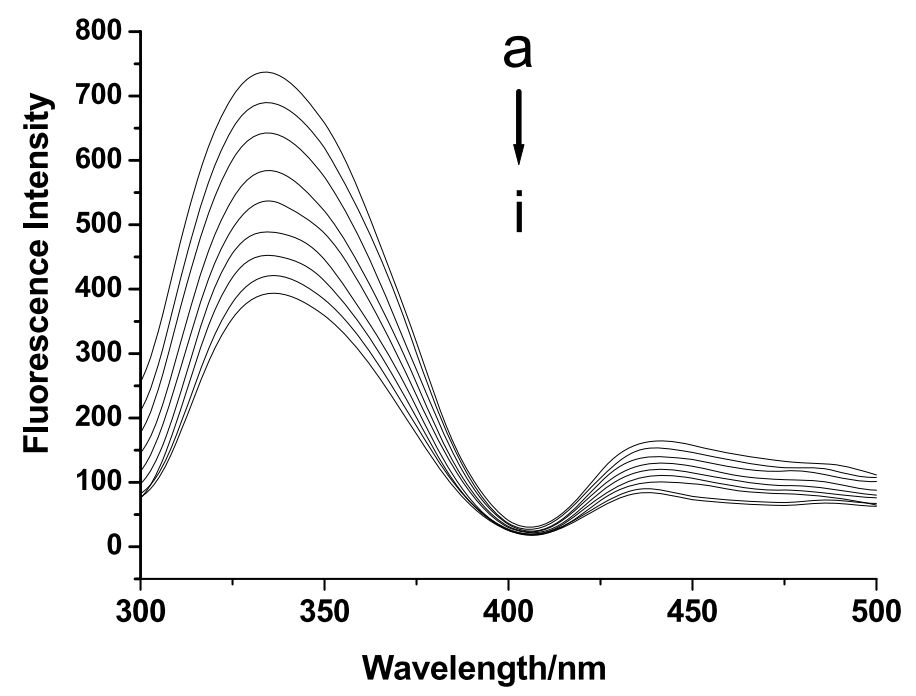

Fig. 1. The fluorescence emission spectra of $5 \mu \mathrm{M} \mathrm{BHb}$ in the presence of TL at $298 \mathrm{~K}$, the concentration of TL from a to $\mathrm{i}$ corresponding to $0 ; 5.0 ; 10.0 ; 15.0 ; 20.0 ; 25.0 ; 30.0 ; 35.0 ; 40.0 \mu \mathrm{M}$.

fluorescence emission peak at $352 \mathrm{~nm}$. Gradual addition of TL to the BHb solution leads to a small bathochromic shift of the emission maximum accompanied by a decrease of fluorescence intensity. It shows that the microenvironment around the fluorophore in the protein is quite different from that of pure one.

The static or dynamic quenching is distinguished by the results at different temperatures. Higher temperature results in faster diffusion and larger amounts of collisional quenching and will typically leads to the dissociation of weakly bound complexes. Therefore, the quenching constant increases with increasing temperature for dynamic quenching. However, it decreases with increase in temperature for static quenching. The possible quenching mechanism can be interpreted by fluorescence quenching spectra of the protein and the Stern-Volmer curves [4] of BHb with TL at 288, 298 and $308 \mathrm{~K}$ as shown in Fig. 2. It can be found that the Stern-Volmer plots were linear and the slopes decreased with increasing temperature, which is consistent with the static type of quenching mechanism. According to Eq. (1),

$$
F_{0} / F=1+K_{\mathrm{q}} \tau_{0}[Q]=1+K_{\mathrm{SV}}[Q],
$$

where $F_{0}$ and $F$ are the fluorescence intensities of BHb in absence and presence of quencher, respectively, $K_{\mathrm{SV}}$ is the Stern-Volmer quenching constant and $[Q]$ is the concentration of quencher (TL). Quenching constant $K_{\mathrm{SV}}$ can be calculated from Fig. 2 and their values are $4.038 \times 10^{4}, 3.557 \times$ $10^{4}, 2.866 \times 10^{4} \mathrm{M}^{-1}$ at 288,298 and $308 \mathrm{~K}$, respectively. These results indicate that the probable quenching mechanism of fluorescence of $\mathrm{BHb}$ by TL is a static quenching process and can predict that a complex formed between TL and $\mathrm{BHb}$.

\subsection{Determinations of binding constants and the number of binding site}

For static quenching, the relationship between fluorescence quenching intensity and the concentration of quenchers can be described by the binding constant formula [2]:

$$
\log \left(F_{0}-F\right) / F=\log K_{\mathrm{A}}+n \log [Q],
$$




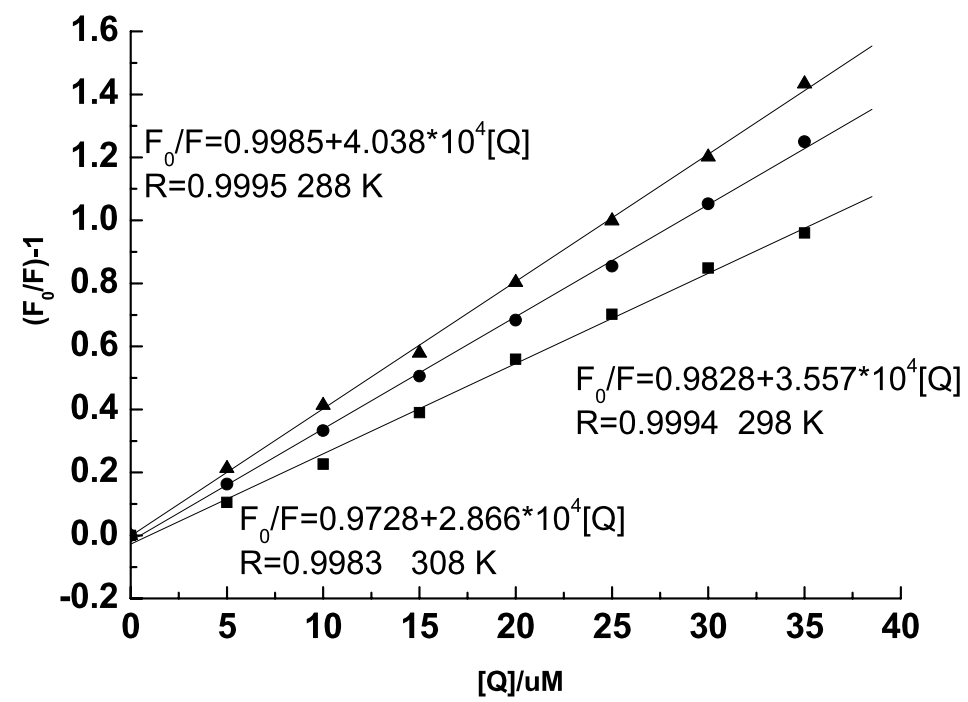

Fig. 2. The Stern-Volmer curves for the binding of TL with BHb at different temperatures. (ム) $288 \mathrm{~K}$; (•) $298 \mathrm{~K}$; $[\mathrm{BHb}]=5.0 \mu \mathrm{M}, \mathrm{pH} 7.4$.

Table 1

Binding constants $K_{\mathrm{A}}$ and binding sites $n$ at different temperatures

\begin{tabular}{llccc}
\hline$T(\mathrm{~K})$ & Linear equation & $R$ & $10^{4} K_{\mathrm{A}}\left(\mathrm{M}^{-1}\right)$ & $n$ \\
\hline 288 & $\log \left[\left(F_{0}-F\right) / F\right]=4.905+1.056 \log [Q]$ & 0.9988 & 8.04 & 1.056 \\
298 & $\log \left[\left(F_{0}-F\right) / F\right]=4.721+1.022 \log [Q]$ & 0.9999 & 5.26 & 1.022 \\
308 & $\log \left[\left(F_{0}-F\right) / F\right]=4.489+0.965 \log [Q]$ & 0.9993 & 3.08 & 0.965 \\
\hline
\end{tabular}

where $K_{\mathrm{A}}$ is the binding constant, and $n$ is the number of binding sites. After the fluorescence quenching intensities were measured, the double-logarithm algorithm was assessed by Eq. (2). The binding constants $\left(K_{\mathrm{A}}\right)$ and the number of binding sites $(n)$ presents in Table 1. The results illustrate that there is a strong binding force between TL and BHb, and the number of binding sites is independent of temperature from 288 to $308 \mathrm{~K}$. The obtained value $n=1.014 \pm 0.02$ indicates that almost one TL binds to a $\mathrm{BHb}$ molecule and it corresponds to the high affinity binding site. It also shows that the values of $K_{\mathrm{A}}$ decrease with the temperature increasing, which is in good agreement with the trend of $K_{\mathrm{SV}}$ as mentioned above.

\subsection{Thermodynamic analysis and determination of the intermolecular forces}

The intermolecular forces between a small molecular and macromolecule mainly include hydrogen bond, van der Waals force, electrostatic force, hydrophobic interaction force and so on. Considering the dependence of binding constant on temperature, a thermodynamic process was considered to be the formation of the complex. Thermodynamic parameters were calculated based on the temperature dependence of the quenching constant for TL-BHb binding. The enthalpy change $\Delta H$ was calculated from the slope of the Van't Hoff equation (3):

$$
\ln K_{\mathrm{SV}}=-\Delta H / R T+\Delta S / R
$$


Table 2

Thermodynamic parameters for CL-BHb complex

\begin{tabular}{lccc}
\hline$T(\mathrm{~K})$ & $\geqslant \Delta G^{0}\left(\mathrm{~kJ} \cdot \mathrm{mol}^{-1}\right)$ & $\geqslant \Delta S^{0}\left(\mathrm{~J} \cdot \mathrm{mol}^{-1} \cdot \mathrm{K}^{-1}\right)$ & $\geqslant \Delta H^{0}\left(\mathrm{~kJ} \cdot \mathrm{mol}^{-1}\right)$ \\
\hline 288 & -27.048 & -25.77 & \\
298 & -26.937 & & -34.54 \\
308 & -26.473 & \\
\hline
\end{tabular}

$K_{\mathrm{SV}}$ is the quenching constant at temperature $T$ and $R$ is a gas constant. The value of $\Delta H$ and $\Delta S$ were obtained from linear Van't Hoff plot. The value of $\Delta G$ was calculated from the Eq. (4):

$$
\Delta G=\Delta H-T \Delta S .
$$

The temperatures chosen were 288, 298 and $308 \mathrm{~K}$. By plotting the quenching constants according to Van't Hoff equation, the thermodynamic parameters were determined from a linear Van't Hoff plot and listed in Table 2. It is clear from the values of standard entropy changes $(\Delta S)$ and standard enthalpy changes $(\Delta H)$ that the binding of TL-BHb is an exothermic process accompanied by a positive $\Delta S$ and a negative $\Delta G$. The binding process is always spontaneous as evidenced by the negative $\Delta G$ value. For drug-protein interaction, Ross and Subramanian have characterized the sign and magnitude of the thermodynamic parameters associated with these various kinds of interactions that play an important role in the protein association process [6]. From the point of their viewpoints, a negative $\Delta H$ and $\Delta S$ value is associated with hydrogen bonding interaction. The negative $\Delta H$ and $\Delta S$ values are associated with hydrogen bonding and van der Waals interaction in low polar dielectric medium. Specific electrostatic interaction between ionic species in an aqueous solution is characterized by positive $\Delta S$ value and very small negative $\Delta H$ value, nearly zero. The negative $\Delta H$ and positive $\Delta S$ values are associated with hydrophobic force and electrostatic force interaction. Thus it is difficult to interpret the thermodynamic parameters of $\mathrm{BHb}-\mathrm{TL}$ interaction with a single intermolecular force. The interaction force between small molecules and proteins is not only a single force in general. May be there are variety of forces existing in the interaction forces, which contains the electrostatic force. It is believed that the hydrophobic small pharmacological molecule cuttage grafting to protein molecules within the hydrophobic region, when a small pharmacological molecule combine with proteins. The solubility of thiacloprid in water is very small, so thiacloprid is belonging to hydrophobic small molecule. It can be cuttage grafting to the internal hydrophobic region of protein molecules with a combination of hydrophobic forces. Therefore, the binding of TL-BHb might involve hydrogen bonding interaction as evidenced by the negative value of $\Delta H$ and $\Delta S$, but hydrophobic force cannot be excluded.

\subsection{CD spectra studies}

To get a full comprehension of how the structure of $\mathrm{BHb}$ is affected by $\mathrm{BHb}, \mathrm{CD}$ measurement was performed on $\mathrm{BHb}$ and the TL-BHb complex. $\mathrm{CD}$ is a powerful method to analysis the secondary structure and tertiary structure. The far UV CD spectrum can give rich information on the change of $\mathrm{BHb}$ conformation, so the secondary structure could be calculated from the data of the far UV CD spectrum, and then the tertiary structure could be distinguished. The $\mathrm{CD}$ spectra of $\mathrm{BHb}$ displays two distinct negative minima in the ultraviolet region, one at $208 \mathrm{~nm}$ and the other at $218 \mathrm{~nm}$, which is the characteristic of $\alpha$-helical structure of a protein. The reasonable explanation is that the negative peak $208 \mathrm{~nm}$ is contributed by $\pi \rightarrow \pi^{*}$ transfer for the peptide bond of $\alpha$-helical, while the negative peak $218 \mathrm{~nm}$ is contributed by $n \rightarrow \pi^{*}$ transfer for the peptide bond of $\alpha$-helical. As can be seen from Fig. 3, the addition 


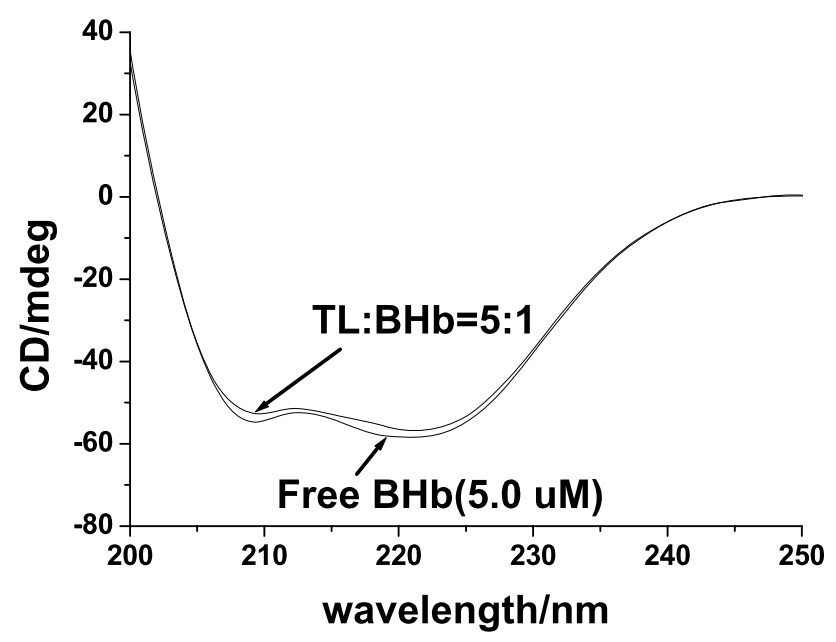

Fig. 3. CD spectra of BHb with and without TL.

of TL-BHb leads to a decrease in the ellipticity without significant shift of the peaks, indicating that the binding of TL-BHb induces a decrease in the $\alpha$-helical content of $\mathrm{BHb}$. However, the CD spectra of $\mathrm{BHb}$ in presence and absence of TL are similar in shape, indicating that the structure of the $\mathrm{BHb}$ is also predominated by $\alpha$-helix after the binding of TL. From the above results, it is apparent that the binding of TL-BHb causes a conformational change of the protein, with the loss of $\alpha$-helix stability. The CD result was expressed as MRE (Mean Residue Ellipticity) in $\operatorname{deg} \mathrm{cm}^{2} \cdot \mathrm{dmol}^{-1}$, which is defined as

$$
M R E=\theta_{\mathrm{obs}} /\left(10 n l C_{\mathrm{p}}\right),
$$

where $\theta_{\text {obs }}$ is the CD in millidegree, $n$ is the number of amino acid residues (574), $l$ is the path length of the cell $(0.1 \mathrm{~cm})$, and $C_{\mathrm{p}}$ is the mole fraction. The $\alpha$-helical contents could be calculated from the MRE values at $209 \mathrm{~nm}$ using the following equation [7]:

$$
\alpha \text {-helical }=\left\{\left(-M R E_{209}-4000\right) /(33000-4000)\right\} \times 100 \text {. }
$$

The results revealed that the content of $\alpha$-helix was decreased from $51.85 \%$ in free $\mathrm{BHb}$ to $48.14 \%$ in TL-BHb complex.

\subsection{Molecule modeling}

The application of molecular modeling has been employed to study the interaction between TL and Bhb. The stereoview of the docking pose of TL with BHb is shown in Fig. 4. As shown in Fig. 4, TL is best located on the surface of the binding pocket of site 2 . The TL is in close proximity to the heme ring and this imply the interference in the oxygen binding property of the protein. The binding free energy $(\Delta G)$ was found to be $-21.34 \mathrm{~kJ} \cdot \mathrm{mol}^{-1}$. Negative value of $\Delta G$ shows that the binding reaction is thermodynamically favorable.

The amino acid residues involved in the binding of TL-Bhb were predicted in Fig. 5 where only residues around $8 \AA$ of TL were displayed. The pyridine ring of TL is making hydrophobic interactions with Leu105, Pro95, Trp37. The interaction between TL and Bhb is not exclusively hydrophobic as 


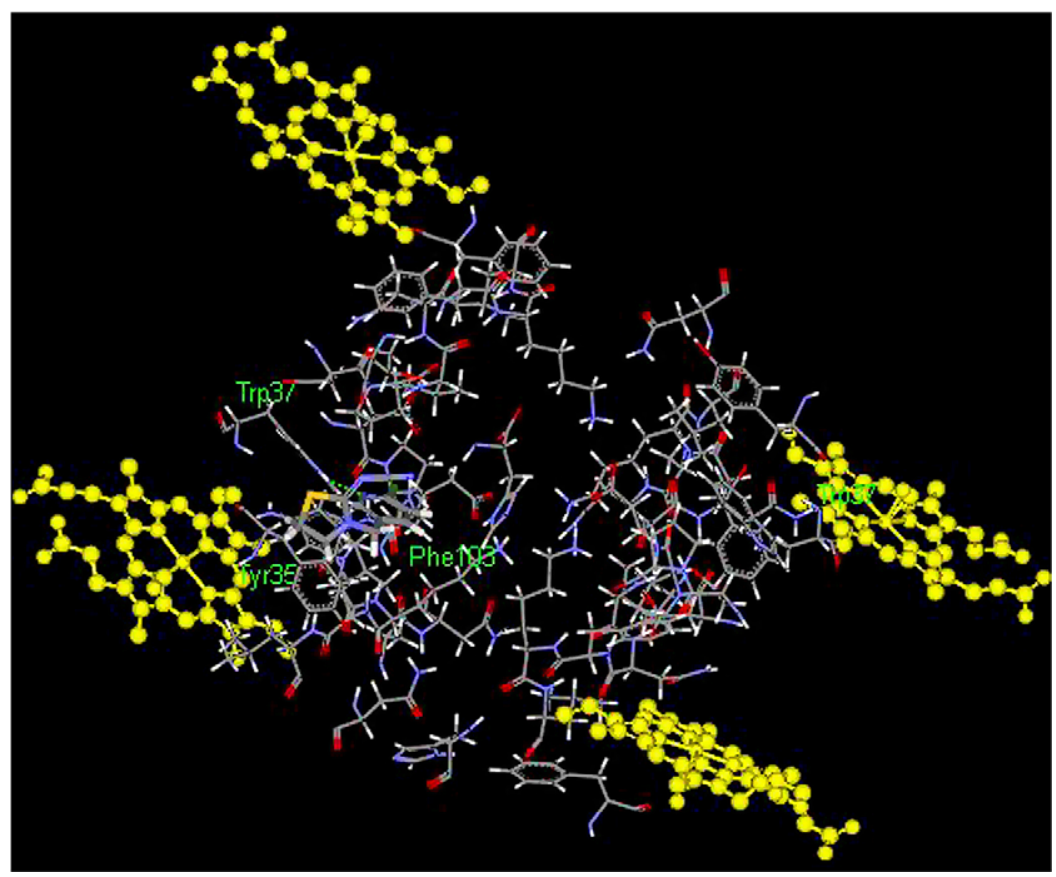

Fig. 4. The stereoview of the docking pose of TL with BHb.

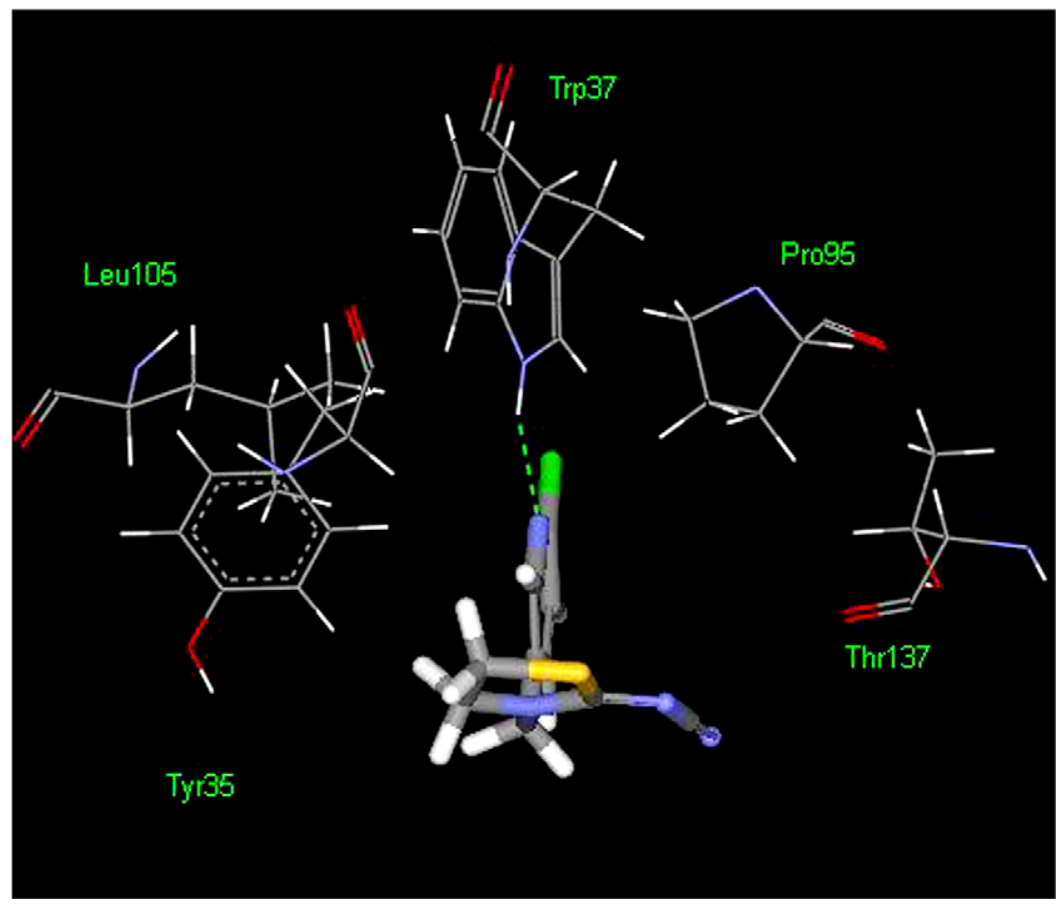

Fig. 5. The amino acid residues involved in the binding of TL-BHb. 
there are several ionic and polar residues (Thr137, Tyr35) in the proximity of bound ligand (within $8 \AA$ ). These polar residues play an inportant role in stabilizing drug via H-bonds and electrostatic interactions. Trp37 is in a suitable position involved in making H-bonds with the imino groups of the side chain. The hydrogen-bonding or electrostatic interaction acts as an anchor and this helps to attain the 3D space position of TL in its biding pocket. This facilitates the hydrophobic interaction of the pyridine rings with the side chain of $\mathrm{BHb}$ residues.

\section{Conclusions}

$\mathrm{BHb}$ was used as a model protein to explore the protein binding properties of TL. The binding of TL-BHb is investigated by fluorescence, circular dichroism spectroscopy and molecular modeling techniques under physiological conditions. The results reveal that the interaction between TL and BHb is a static process. The hydrogen bonding and hydrophobic interaction play a major role during the binding. The conformational alternations are investigated by $\mathrm{CD}$ and the molecular modeling was also used to explore the binding mechanism of TL-BHb.

\section{Acknowledgements}

This work was supported by the Nanometer Technology Special Project (No. 0852nm06200), and the Technology Standard Project (No. 08DZ0505000) of Shanghai Municipal Science and Technology Commission and the National Natural Science Foundation of China (20676051).

\section{References}

[1] X.Y. Bao, Z.W. Zhu, N.Q. Li and J.G. Chen, Electrochemical studies of rutin interacting with hemoglobin and determination of hemoglobin, Talanta 54(4) (2001), 591-596.

[2] C.Y. Chen, K. Chen, Q. Long, M.H. Ma and F. Ding, Structural characterization and DNA-binding properties of Sm(III) complex with ofloxacin using spectroscopic methods, Spectroscopy 23 (2009), 103-111.

[3] C.Y. Chen, J.H. Zhou and X.T. Gu, Binding studies of paeonolum with bovine serum albumin using spectroscopic methods, Spectroscopy 21 (2007), 53-60.

[4] Y.J. Hu, Y. Liu, R.M. Zhao et al., Spectroscopic studies on the interaction between methylene blue and bovine serum albumin, J. Photoc. Photob. A 179 (2006), 324-329.

[5] R. Osterauer and H.-R. Köhler, Temperature-dependent effects of the pesticides thiacloprid and diazinon on the embryonic development of zebrafish (Danio rerio), Aquat. Toxicol. 86 (2008), 485-494.

[6] P.D. Ross and S. Subramanian, Thermodynamics of protein association reactions: forces contributing to stability, Biochemistry 20 (1981), 3096-3102.

[7] S. Sugio, A. Kashima, S. Mochizuki, M. Noda and K. Kobayashi, Crystal structure of human serum albumin at $2.5 \AA$ resolution, Protein Eng. 12 (1999), 439-446. 


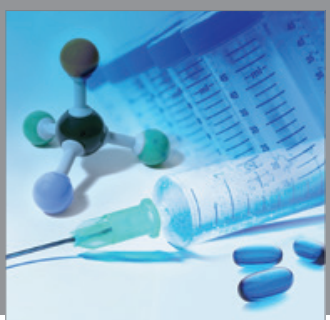

International Journal of

Medicinal Chemistry

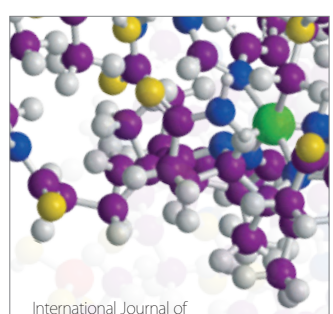

Carbohydrate Chemistry

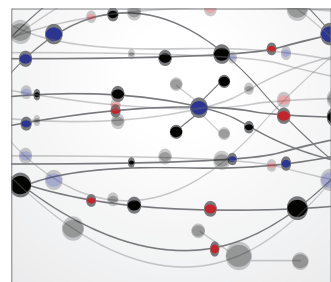

The Scientific World Journal
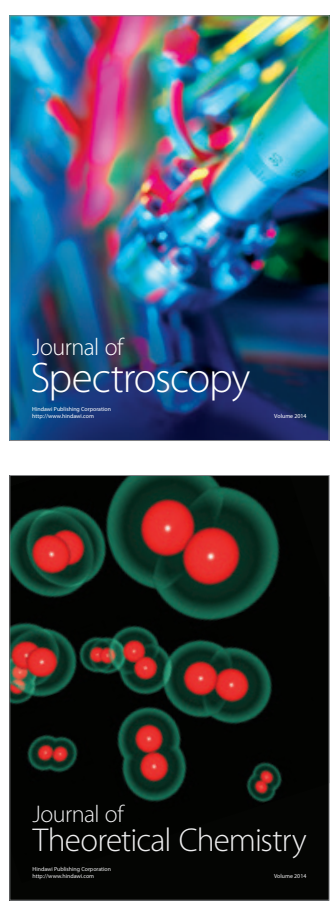
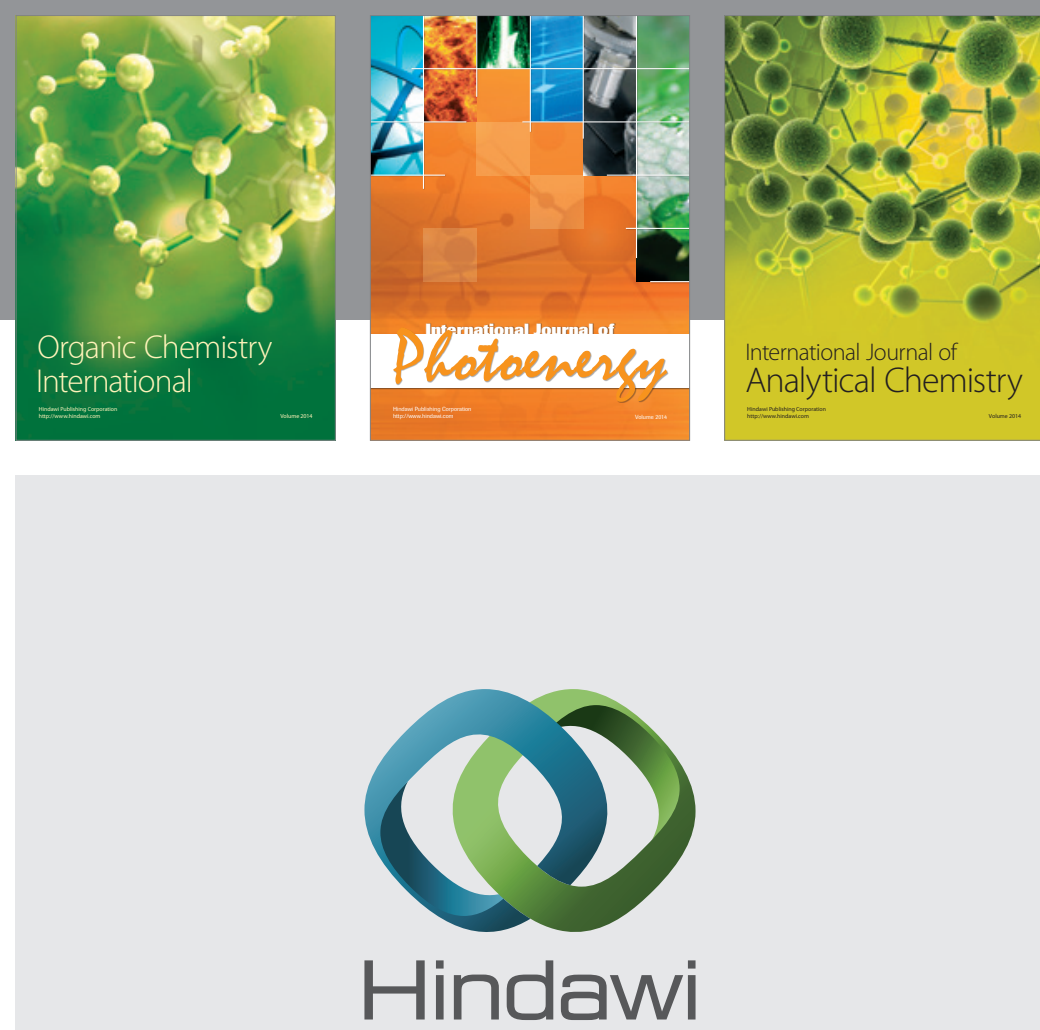

Submit your manuscripts at

http://www.hindawi.com
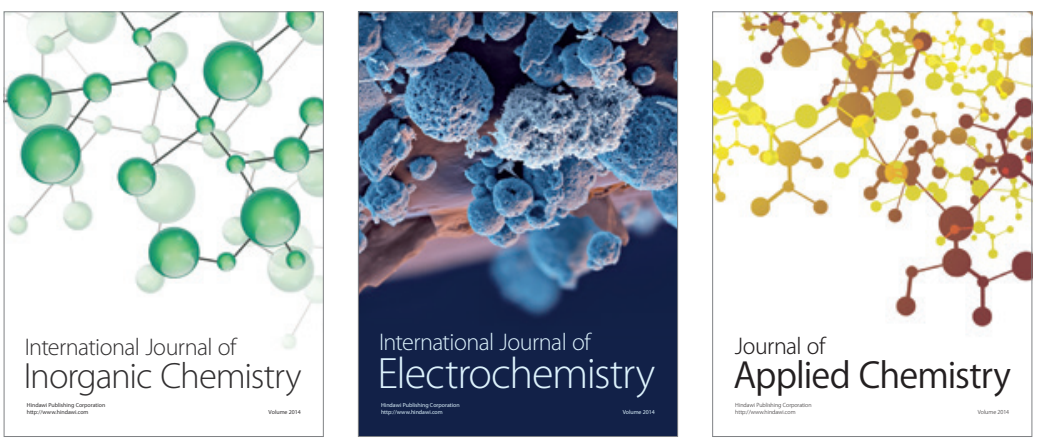

Journal of

Applied Chemistry
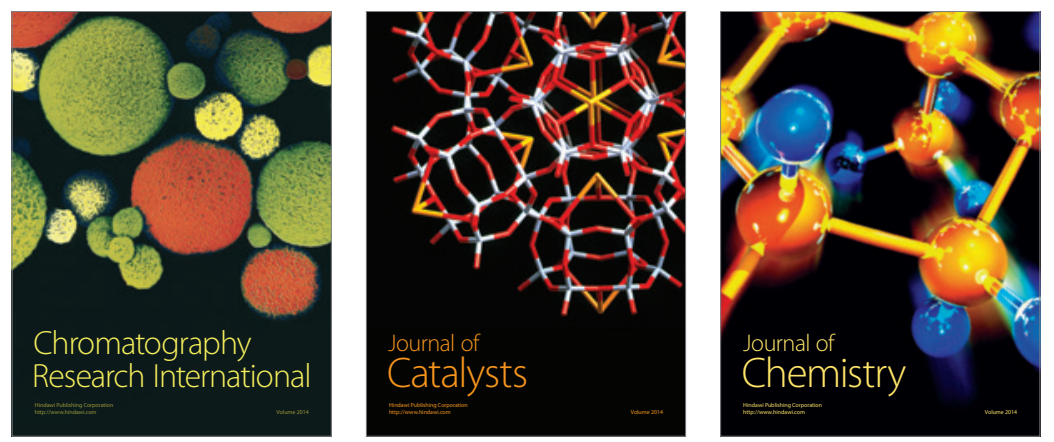
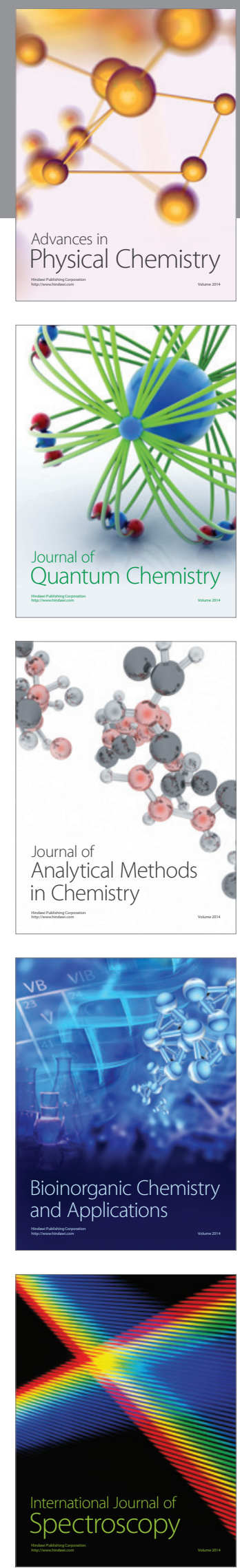